\title{
The efficacy of fosfomycin trometamol in prevention of postoperative urinary tract infection in pregnant women after endoscopic procedures: open clinical trial
}

\author{
Mohamed Radwan ${ }^{1}$, Ayman Hagras', Salah Nagla ${ }^{1 *} \mathbb{C}$, Nareman Elhamamy ${ }^{2}$ and Mohammed Abou Farha ${ }^{1}$
}

\begin{abstract}
Background: Fosfomycin tromethamine is recommended (category B) for the treatment of urinary tract infections in pregnant women. We aimed to evaluate the efficacy of single-dose preoperative fosfomycin tromethamine in the prevention of urinary tract infection in pregnant women who underwent lower urinary tract endoscopic surgeries.

Methodology: The subjects of this study were pregnant women who underwent lower urinary tract endoscopic procedures. All patients received Fosfomycin tromethamine1 packet $(3 \mathrm{~g})$ dissolved in water the day before surgery. Routine pre- and postoperative urine analysis and urine cultures were performed when needed. Patients were monitored for urinary tract infections and the side effects of the drug. Moreover, maternal and fetal complications were monitored.

Results: The study was conducted on 31 pregnant women with a mean age of $24.48 \pm 5.35$ years. The endoscopic procedures were as follows: 28 (90.3\%) DJ ureteric insertions for hydronephrosis, 2 (6.5\%) urinary bladder stones, and cystoscopy (3.2\%) for gross hematuria. Two (6.5\%) patients had postoperative asymptomatic bacteriuria that resolved with oral antibiotic therapy.

Conclusion: Fosfomycin tromethamine is safe in preventing urinary tract infection and decreases the need for postoperative parenteral antibiotics in pregnant women who require lower urinary tract endoscopic procedures.
\end{abstract}

Keywords: Fosfomycin, Pregnancy, UTI

\section{Background}

Urinary tract infection (UTI) is the most common cause of hospital admission in pregnant women [1]. UTIs may complicate up to $10 \%$ of pregnancies, which may threaten pregnancy and would have negative effects on pregnant women [2]. Fosfomycin trometamol is an FDAapproved drug for pregnant women (category B) with a wide spectrum of antimicrobial activity against common uropathogens $[3,4]$. Fosfomycin trometamol is usually administered in a single oral dose to reach a high urinary concentration and can be prescribed as prophylaxis prior to many endoscopic procedures $[4,5]$. In this study, we evaluated the efficacy of one dose of Fosfomycin trometamol ( $3 \mathrm{~g})$ orally to prevent postoperative urinary tract infection and the postoperative need for parenteral antibiotics in pregnant women after lower urinary tract endoscopic procedures. To the best of our knowledge, this is the first study to discuss this issue in pregnant women.

\footnotetext{
*Correspondence: salahnaglah@yahoo.com

1 Urology Department, Tanta University, Tanta, Egypt

Full list of author information is available at the end of the article
} 


\section{Methods}

This prospective, clinical trial was conducted at both departments of Urology and department of Obstetrics and Gynecology in the period from February 2016 to January 2020.

\subsection{Recruitment}

Thirty-one patients between the second and third trimesters were enrolled in this study. All patients underwent lower urinary tract endoscopic procedures. The exclusion criteria were as follows: (a) immunocompromised patients, for example, diabetes mellitus; (b) patients with signs of pyelonephritis, for example, fever associated with flank pain; (c) patients unable to receive oral medication, such as severe nausea and/or vomiting; (d) patients sensitive to the drug; and (e) patients who needed upper urinary tract endoscopic surgeries.

\subsection{Dosage and administration}

Fosfomycin tromethamine sachet was dissolved in a cup of water and taken the night before the operation at bedtime.

\subsection{Procedures}

All patients' age, parity, gestational age, and symptoms in addition to abdomen/pelvis ultrasonography and mid-stream urine analysis were recorded at the beginning of the study. Patients with genital tract infections were treated before the procedure. Asymptomatic bacteriuria ( $>100,000 \mathrm{CFU} / \mathrm{ml}$, no symptoms of infection) and symptomatic UTIs (urine culture $>10,000 \mathrm{CFU} /$ $\mathrm{ml}$ with symptoms, dysuria, urinary frequency, and urgency) were treated and recorded before endoscopic surgery. All endoscopic procedures were performed under general intubated anesthesia. Maintenance of stable hemodynamics and average oxygen saturation were monitored by the anesthesiologist during the procedure and in the recovery room after surgery. The patients were placed in the lithotomy position. All the procedures were performed under complete sterilization. A urinary indwelling catheter was left only one day after the endoscopy. Complete urine analysis was performed after the catheter was removed. Patients were then followed up by the 4th author for prematurity and maternal and fetal complications.

\subsection{Statistical analysis}

The Statistical Package for Social Sciences (SPSS; version 21) for the analysis. The statistical methods used were descriptive statistics, frequency analysis, mean, standard deviation, and percentage.

\subsection{The primary outcome}

was the presence of urinary tract infection, whether asymptomatic bacteriuria (no symptoms of infection, $>100,000 \mathrm{CFU} / \mathrm{ml}$ ), and symptomatic UTIs (with symptoms such as dysuria and urgency, urine culture $>10,000 \mathrm{CFU} / \mathrm{ml}$ ). The secondary outcome was the presence of side effects to the drug and the presence of complications to the mother and fetus, for example, premature delivery.

\section{Results}

The study included 31 women with a mean age of $24.48 \pm 5.35$ years. Twenty-seven $(87.1 \%)$ patients were in their second trimester, while four (12.9\%) were in their third trimester (Table 1). The endoscopic procedures were as follows: $28(90.3 \%)$ DJ ureteric insertions for hydronephrosis, 2 (6.5\%) urinary bladder stones, and cystoscopy (3.2\%) for gross hematuria. Our mean operative time was $24.2 \pm 4.5$ minutes. Side effects were reported in 3 (9.7\%) patients, 2(6.5\%) had diarrhea, and 2(6.5\%) had nausea and vomiting. Two patients (6.5\%) had asymptomatic bacteriuria that responded to oral antibiotics.

\section{Discussion}

Fosfomycin is a broad-spectrum antibiotic for both gram-negative and gram-positive bacteria. A single dose of fosfomycin tromethamine produces a therapeutic concentration in the urine for 1-3 days, and fosfomycin concentration in urine is maintained at high levels for 2-3 days $[6,7]$. Comparative studies have shown that a single 3-g dose of fosfomycin tromethamine is comparable to a one-week treatment regimen of standard antibiotics such as nitrofurantoin and co-trimoxazole in the treatment of UTIs. Moreover, fosfomycin is recommended for the treatment of urinary tract infection during pregnancy (FDA-approved category B) with comparable results and tolerable side effects [8]. Usta et al. [9] treated 324 pregnant women with lower urinary tract infections

Table 1 Number of gravidity and correlation to the trimester

\begin{tabular}{lrrrr}
\hline & & \multicolumn{2}{l}{ Trimester } & Total \\
\cline { 3 - 4 } & & $\mathbf{2}$ & $\mathbf{3}$ & \\
\hline Gravidity & 1 & 19 & 1 & 20 \\
& 2 & 5 & 2 & 7 \\
& 3 & 2 & 1 & 3 \\
Total & 4 & 1 & 0 & 1 \\
\hline
\end{tabular}


and randomized them into three groups. The groups did not differ in terms of cure rates or side effects. They concluded that treatment with a single dose of fosfomycin trometamol is as effective as the treatment course with cefuroxime axetil or amoxicillin-clavulanic. In addition, fosfomycin trometamol showed better compliance. In our study, patients received a single dose of fosfomycin $3 \mathrm{~g}$ the night before the operation. Patients were followed up for the presence of urinary tract infections and the occurrence of drug side effects. Fosfomycin has been evaluated for use in many endourologic interventions in adults. At the same time, there are not many data about the use of single-dose fosfomycin in endourological interventions in pregnant women. In their meta-analysis, Noreikaite et al. [10] concluded that fosfomycin has significantly lower septic complications with an equivalent side effect profile compared to the quinolone-based prophylaxis regimen for transrectal ultrasound-guided prostate biopsy. In addition, Besien et al. [11] switched ciprofloxacin to fosfomycin in the case of fluoroquinolone-resistant rectal bacteria. They concluded that fosfomycin showed promising results in patients with fluoroquinolone resistance. Moreover, Yang et al. [12] proved that fosfomycin is comparable to parenteral cefoxitin sodium in the prevention of urinary tract infection after transurethral resection of bladder tumors. In addition, Khawaja et al. [5] evaluated the role of prophylactic single-dose fosfomycin tromethamine in 72 endourologic procedures. Five days later, only eight patients had asymptomatic bacteriuria. In our study, all of our patients were symptomatically free with no urinary tract infection, as documented by postoperative urine analysis. Regarding complications, most of the reported side effects in the literature were gastrointestinal upsets [13-15]. Khawaja et al. [5] reported that the side effects were transient and subsided without any medication. The most reported side effects were diarrhea and genital itching. In our study, 2(6.5\%) patients had asymptomatic bacteriuria that responded to oral antibiotic therapy. None of the patients required postoperative parenteral antibiotics. Side effects were reported in $3(9.7 \%)$ patients, 2(6.5\%) had diarrhea, and $2(6.5 \%)$ had nausea and vomiting. The side effects were mild, tolerable, and subsided with medical therapy. We did not record the need for re-hospitalization to treat any drug-related side effects. To our knowledge, this is the first study to address the results of fosfomycin tromethamine as a prophylactic therapy in pregnant women who undergo lower urinary tract endoscopic procedures.

\section{Study limitations}

A control arm is lacking in this study. Study has a small number of patients.

\section{Conclusions}

Fosfomycin tromethamine is safe in preventing urinary tract infection and decreases the need for postoperative parenteral antibiotics in pregnant women who require lower urinary tract endoscopic procedures.

\section{Abbreviations}

UTI: Urinary tract infection; FDA: United states food and drug administration; DJ: Double J.

\section{Authors' contributions}

MR organized and designed the study. $\mathrm{AH}, \mathrm{NH}$, and SN drafted the manuscript and performed the statistical analysis. MF was responsible for the clinical assessment of the subjects and, together with MR, contributed in the interpretation of the results. All authors contributed in writing of the manuscript. All authors have read and agreed the final version of the manuscript.

\section{Funding}

We are self-funded.

Availability of data and materials

It is available when asked from the corresponding author after approval from our institute.

\section{Declarations}

\section{Ethics approval and consent to participate}

were obtained before the start of treatment by Tanta university ethical committee. An informed consent was obtained from all the subjects of the study before enrollment. The study has been performed in accordance with the 1964 Declaration of Helsinki and its later corrections, or comparable ethical standards. The drug, procedures, and their possible complications were discussed in detail with all patients, and an informed consent was signed from the subjects of this study before the beginning of the procedures. Patients' confidentiality was maintained throughout this study.

\section{Competing interests}

The author declare that they have no competing interests.

\section{Consent for publication}

(Not applicable) Manuscript does not contain data from any individual person.

\section{Author details}

${ }^{1}$ Urology Department, Tanta University, Tanta, Egypt. ${ }^{2}$ Obstetrics and Gynecology Department, Tanta University, Tanta, Egypt.

Received: 27 September 2021 Accepted: 6 December 2021

Published online: 24 December 2021

\section{References}

1. Colgan R, Williams M (2011) Diagnosis and treatment of acute uncomplicated cystitis. Am Fam Phys 84(7):771-776 (PMID: 22010614)

2. Hooton TM (2012) Clinical practice. Uncomplicated urinary tract infection N Engl J Med. 366(11): 1028-37. https://doi.org/10.1056/NEJMcp1104429

3. Falagas M, Kastoris A, Kapaskelis A, Karageorgopoulos D (2010) Fosfomycin for the treatment of multidrug-resistant, including extendedspectrum beta-lactamase producing Enterobacteriaceae infections: a systemic review. Lancet Infect Dis 10:43-50. https://doi.org/10.1016/ S1473-3099(09)70325-1

4. Lob S, Nicolle L, Hoban D, Kazmierczak K, Badal R, Sahm D (2016) Susceptibility patterns and ESBL rates of Escherichia from urinary tract infections in Canada and the United States, SMART 2010-2014. Diagn Microbiol Infect Dis 85:459-465. https://doi.org/10.1016/j.diagmicrobio.2016.04.022 
5. Khawaja A, Khan F, Dar T, Bhat A, Wani M, Wazir B (2015) Fosfomycin tromethamine antibiotic of choice in the female patient: a multicenter study. Cent Eur J Urol 68:371-375. https://doi.org/10.5173/ceju.2015.590

6. Song F, Liu C, Zhang J et al (2020) Antibacterial effect of fosfomycin tromethamine on the bacteria inside urinary infection stones. Int Urol Nephrol 52(4):645-654. https://doi.org/10.1007/s1 1255-019-02358-0

7. Wang T, Wu G, Wang J et al (2020) Comparison of single-dose fosfomycin tromethamine and other antibiotics for lower uncomplicated urinary tract infection in women and asymptomatic bacteriuria in pregnant women: a systematic review and meta-analysis. Int J Antimicrob Agents 56(1):106018. https://doi.org/10.1016/j.ijantimicag.2020.106018

8. Dawood AS, Dawood AS, Nagla SA, El-Bakary MA (2017) Fosfomycin therapy for non-complicated lower urinary tract infections during pregnancy: tanta university experience. Open J Obstetr Gynecol 7:532-544. https://doi.org/10.4236/ojog.2017.75056

9. Usta TA, Dogan O, Ates U, Yucel B, Onar Z, Kaya E (2011) Comparison of single-dose and multiple-dose antibiotics for lower urinary tract infection in pregnancy. Int J Gynaecol Obstet 114:229-233. https://doi.org/10. 1016/j.ijgo.2011.03.014

10. Noreikaite J, Jones P, Fitzpatrick J, Amitharaj R, Pietropaolo A, Vasdev N, et al (2018) Fosfomycin vs. quinolone-based antibiotic prophylaxis for transrectal ultrasound-guided biopsy of the prostate: a systematic review and meta-analysis. Prostate Cancer Prostatic Dis. 21 (2): 153-160. https:// doi.org/10.1038/s41391-018-0032-2

11. Van Besien J, Uvin P, Weyne E, Van Praet C, Merckx L, De Graeve N et al (2019) Use of fosfomycin as targeted antibiotic prophylaxis before prostate biopsy: a prospective randomized study. Int J Urol 26(3):391-397. https://doi.org/10.1111/iju.13883

12. Yang J, Zhang P, Zhou H, Feng S, Zhang X (2018) Prospective, randomized controlled study of the preventive effect of fosfomycin tromethamine on post-transurethral resection of bladder tumor urinary tract infection. Int J Urol. 25(10): 894-895. https://doi.org/10.1111/iju.13719

13. Keating GM (2013) Fosfomycin trometamol: a review of its use as a single dose oral treatment for patients with acute lower urinary tract infections and pregnant women with asymptomatic bacteriuria. Drugs 73:19511966. https://doi.org/10.1007/s40265-013-0143-y

14. Ceran N, Mert D, Kocdogan FY, Erdem I, Adalati R, Ozyurek S et al (2010) A randomized comparative study of single-dose fosfomycin and 5-day ciprofloxacin in female patients with uncomplicated lower urinary tract infections. J Infect Chemother 16:424-430. https://doi.org/10.1007/ s10156-010-0079-z

15. Konwar M, Gogtay NJ, Ravi R, Thatte UM, Bose D (2021) Evaluation of efficacy and safety of fosfomycin versus nitrofurantoin for the treatment of uncomplicated lower urinary tract infection (UTI) in women-A systematic review and meta-analysis. J Chemother. 21: 1-10 https://doi.org/10.1080/ 1120009X.2021.1938949.

\section{Publisher's Note}

Springer Nature remains neutral with regard to jurisdictional claims in published maps and institutional affiliations.

\section{Submit your manuscript to a SpringerOpen ${ }^{\circ}$ journal and benefit from:}

- Convenient online submission

- Rigorous peer review

- Open access: articles freely available online

- High visibility within the field

- Retaining the copyright to your article

Submit your next manuscript at $\boldsymbol{\nabla}$ springeropen.com 